\title{
Comparison of Ocular Biometry and Refractive Outcomes Using IOL Master 500, IOL Master 700, and Lenstar LS900
}

\author{
Jae Shin Song ${ }^{1}$, Do Yeh Yoon ${ }^{1,2}$, Joon Young Hyon ${ }^{1,2}$, Hyun Sun Jeon ${ }^{1,2}$ \\ ${ }^{1}$ Department of Ophthalmology, Seoul National University College of Medicine, Seoul, Korea \\ ${ }^{2}$ Department of Ophthalmology, Seoul National University Bundang Hospital, Seongnam, Korea
}

Purpose: To evaluate the agreement in ocular biometry outcomes measured by three different devices, the IOL Master 500, IOL Master 700, and Lenstar LS900, and compare the refractive outcomes after cataract surgery obtained using those three devices.

Methods: Medical records of 178 eyes of 89 patients who underwent ocular biometry with the three devices were retrospectively reviewed, and 124 eyes met the inclusion criteria. Paired comparisons were performed for axial length (AL), mean keratometry $(\mathrm{Km})$, and anterior chamber depth and quantified their agreement using Bland-Altman plots. Subgroup analyses were done according to the $\mathrm{AL}$ and the $\mathrm{Km}$. Refractive outcomes were compared with respect to absolute prediction errors after cataract surgery in 54 eyes.

Results: Among 124 eyes, 12, 3, and 5 eyes failed to be measured of AL by IOL Master 500, IOL Master 700 and Lenstar LS900, respectively. The AL measured by Lenstar LS900 was longer than that measured by IOL Master 500 and IOL Master 700 ( $p<0.001, p=0.002$, respectively). Subgroup analysis revealed that these results were statistically significant only in long eyes $(A L>25.5 \mathrm{~mm}$ ). $\mathrm{Km}$ measured using the IOL Master 500 was steeper than that measured with the IOL Master 700 or Lenstar LS900 ( $p=0.001, p<0.001$, respectively). anterior chamber depth measured by IOL Master 500 was shallower than that measured by IOL Master 700 or Lenstar LS900 ( $p<0.001, p<0.001$, respectively). Ocular biometry measurements by the three devices showed high agreement with narrow $95 \%$ limits of agreement. Absolute prediction errors from the 3 devices showed no statistically significant differences after cataract surgery.

Conclusions: The IOL Master 700 and Lenstar LS900 demonstrated superior acquisition rates of biometric measurements compared with the IOL Master 500. Ocular biometry using the 3 different devices showed high agreement, although statistically significant differences were observed; however, since there was no difference in predicting the refractive outcomes, those differences are clinically negligible.

Key Words: Biometry, Cataract, Low coherence reflectometry, Swept source optical coherence tomography

Received: August 18, 2019 Final revision: November 12, 2019 Accepted: November 13, 2019

Corresponding Author: Hyun Sun Jeon, MD. Department of Ophthalmology, Seoul National University Bundang Hospital, 82 Gumi-ro 173beon-gil, Bundang-gu, Seongnam 13620, Korea. Tel: 82-31-787-7383, Fax: 82-31-787-4057, E-mail: jeonhs@snu.ac.kr
Satisfaction of patients undergoing cataract surgery is dependent on precise predictions of refractive outcomes. As accurate biometry and power precision of intraocular lens (IOL) have gained importance, development of various modern diagnostic techniques has enabled the control 
of refractive outcomes with ever-increasing accuracy [1].

IOL Master 500 (Carl Zeiss Meditec AG, Jena, Germany), which uses the principle of partial coherence interferometry, has become the standard technique for its high accuracy and similar results to those achieved by ultrasound biometry [2,3]. However, in cases of severe lens opacity, measurements may not be obtained, and in cases of poor visual acuity, infrared rays may be reflected on other parts than the fovea, resulting in inaccurate measurements. Recently, newer instruments have been introduced to compensate for these drawbacks. The IOL Master 700 (Carl Zeiss Meditec AG) is based on the principle of swept source optical coherence tomography (SS-OCT), and it enables visualization of the complete longitudinal section of the eye [4]. Therefore, imaging of the fovea has made it possible to monitor for poor fixation during measurement. The Lenstar LS900 (Haag Steit AG, Koeniz, Switzerland) is a newly developed biometry device that uses the principle of low-coherence reflectometry. From a single measurement, approximately 20 ocular biometry parameters can be measured simultaneously, and multiple measurements can be taken sequentially to improve accuracy [5].

There is a paucity of data comparing the ocular biometry and refractory outcomes among the 3 devices: IOL Master 500, IOL Master 700, and Lenstar LS900. The purpose of this study was to evaluate the agreement of ocular biometry outcomes measured using the three different devices and to compare the refractory outcomes after cataract surgery obtained by those three devices.

\section{Materials and Methods}

\section{Patients and methods}

This study protocol adhered to the tenets of the Declaration of Helsinki and was approved by the institutional review board of Seoul National University Bundang Hospital (B-1812-508-101). Informed consent was waived due to the retrospective nature of the study.

We performed a retrospective study with medical records of 178 eyes in 89 patients who underwent ocular biometry measurements before cataract surgery by all three devices, the IOL Master 500, IOL Master 700, and Lenstar LS900, at a single tertiary institution (Seoul National University Bundang Hospital) from June 2018 to August 2018 for inclusion in the present study. Exclusion criteria were eyes with retinopathy or maculopathy, eyes with history of corneal disease, eyes that underwent previous ocular surgery, eyes with history of ocular trauma, and pseudophakic eyes. Finally, 112 eyes fulfilled the inclusion criteria. Of the 178 eyes with ocular biometry measurements available with the three devices, 54 eyes were excluded and 12 additional eyes were excluded due to failure of axial length (AL) measurement; thus, 112 eyes were analyzed for comparison.

Of the 112 eyes, 54 eyes that had implanted monofocal aspheric IOLs, ZCB00 (Tecnis; AMO, Santa Ana, CA, USA) during cataract surgery were analyzed to compare refractive outcomes after surgery.

\section{Main measurement outcomes}

$\mathrm{AL}$, mean keratometry $(\mathrm{Km})$, and anterior chamber depth (ACD), all of which are crucial for IOL power calculation, were adopted as the main measurement outcomes for comparison [6]. Refractive outcomes were compared by absolute prediction error (PE), which was performed 4 weeks after cataract surgery. Absolute PE was calculated as absolute value of the refractive PE, which was calculated as postoperative spherical equivalent (SE, combination of the sphere and the $1 / 2$ cylinder) minus predicted postoperative SE. We applied the distinct formulae in the built-in software of each device: the Haigis formula for the IOL Master 500, the SRK/T for the IOL Master 700, and the Hill-RBF for the Lenstar LS900.

\section{Statistical analysis}

Statistical analyses were performed using IBM SPSS Statistics ver. 22.0 (IBM Corp., Armonk, NY, USA). The paired $t$-test was used to establish whether there was a significant systematic bias among the three devices. Bland-Altman plots were used to graphically present agreement between two different devices. A $p$-value less than 0.05 was considered statistically significant.

\section{Results}

Of the 178 eyes with ocular biometry measurements available with the three devices, 124 eyes met the inclusion 
criteria, and 12 additional eyes (9.7\%) were excluded due to failure of AL measurement; thus, a total of 112 eyes (mean age, $65 \pm 10$; range, 41 to 91 years) were finally analyzed. Of the 12 eyes that failed the AL measurement, all 12 failed to be measured by the IOL Master 500, while three eyes (2.4\%) and five eyes (4.0\%) failed with the IOL Master 700 and Lenstar LS900, respectively. All 12 of the failed eyes were from patients over 70 years old whose nuclear scores by Lens Opacities Classification System (LOCS) III [7] were over 3, except for three eyes with posterior subcapsular cataracts (PSC). The mean age of the patients with the 12 eyes that failed measurement by IOL Master 500 was $72 \pm 3$ years, while that of the five eyes that failed by Lenstar LS900 was $76 \pm 3$ years and that of the three eyes that failed by IOL Master 700 was $77 \pm 4$ years. Among the four eyes with PSC that failed measure- ment by IOL Master 500, three eyes were measurable with the IOL Master 700 and two eyes were measurable with the Lenstar LS900.

The mean biometric measurements with the three optical biometry devices are shown in Table 1 . The mean AL measurement obtained using the Lenstar LS900 was significantly longer than that obtained with the other two devices, the IOL Master 500 and the IOL Master 700, by mean differences of $0.012 \pm 0.03 \mathrm{~mm}(p<0.001)$ and 0.012 $\pm 0.04 \mathrm{~mm}(p=0.002)$, respectively. The mean Km obtained with the IOL Master 500 was significantly higher than that from the IOL Master 700 and Lenstar LS900, respectively ( $p<0.001$ and $p<0.001$, respectively). The mean ACD obtained with the IOL Master 500 was significantly shorter than the IOL Master 700 and Lenstar LS900 ( $p=0.001$ and $p<0.001$, respectively).

Table 1. Biometry measurements by IOL Master 500, IOL Master 700, and Lenstar LS900

\begin{tabular}{lccc}
\hline Parameter & IOL Master 500 & IOL Master 700 & Lenstar LS900 \\
\hline AL $(\mathrm{mm})$ & $24.12 \pm 1.74(0.858,<0.001)$ & $24.12 \pm 1.73(0.858,0.002)$ & $24.13 \pm 1.75^{*}(<0.001,0.002)$ \\
Km $(\mathrm{D})$ & $44.23 \pm 1.48^{*}(0.001,<0.001)$ & $44.12 \pm 1.51(0.001,0.237)$ & $44.16 \pm 1.49(<0.001,0.237)$ \\
ACD $(\mathrm{mm})$ & $3.04 \pm 0.46^{*}(<0.001,<0.001)$ & $3.15 \pm 0.48(<0.001,0.002)$ & $3.16 \pm 0.44(<0.001,0.002)$ \\
\hline
\end{tabular}

Values are presented as mean \pm standard deviation ( $p$-value, compared with the other two devices in order of IOL Master 500, IOL Master 700 and Lenstar LS900).

$\mathrm{AL}=$ axial length; $\mathrm{Km}=$ mean keratometry; $\mathrm{D}=$ diopters; $\mathrm{ACD}=$ anterior chamber depth.

"Significantly different at $p<0.05$.

Table 2. Subgroup analysis for biometry measurements by axial length

\begin{tabular}{lcccc}
\hline Axial length subgroup & Number & \multicolumn{1}{c}{ IOL Master 500 } & IOL Master 700 & Lenstar LS900 \\
\hline Long $(>25.5 \mathrm{~mm})$ & 17 & $27.48 \pm 1.40(0.729,<0.001)$ & $27.48 \pm 1.38(0.729,0.002)$ & $27.53 \pm 1.41^{*}(<0.001,0.002)$ \\
Medium $(22.5-25.5 \mathrm{~mm})$ & 80 & $23.76 \pm 0.76(0.559,0.056)$ & $23.76 \pm 0.76(0.559,0.262)$ & $23.77 \pm 0.76(0.056,0.262)$ \\
Short $(<22.5 \mathrm{~mm})$ & 15 & $22.22 \pm 0.27(0.845,0.433)$ & $22.21 \pm 0.26(0.845,0.066)$ & $22.22 \pm 0.27(0.433,0.066)$ \\
\hline
\end{tabular}

Values are presented as mean \pm standard deviation ( $p$-value, compared with the other two devices in order of IOL Master 500, IOL Master 700, and Lenstar LS900).

*Significantly different at $p<0.05$.

Table 3. Subgroup analysis for biometry measurements by mean keratometry

\begin{tabular}{lcccc}
\hline Keratometry subgroup & Number & IOL Master 500 & IOL Master 700 & Lenstar LS900 \\
\hline Steep (>46 D) & 15 & $46.36 \pm 0.22(0.146,0.057)$ & $46.05 \pm 0.88(0.146,0.347)$ & $46.25 \pm 0.34(0.057,0.347)$ \\
Medium (42-46 D) & 90 & $44.09 \pm 1.15^{*}(0.003,<0.001)$ & $44.03 \pm 1.20(0.003,0.875)$ & $44.03 \pm 1.17(<0.001,0.875)$ \\
Flat (<42 D) & 7 & $41.45 \pm 0.51(0.068,0.207)$ & $41.28 \pm 0.38(0.068,0.382)$ & $41.33 \pm 0.44(0.207,0.382)$ \\
\hline
\end{tabular}

Values are presented as mean \pm standard deviation ( $p$-value, compared to the other two devices in order of IOL Master 500, IOL Master 700, and Lenstar LS900).

$\mathrm{D}=$ diopters.

*Significantly different at $p<0.05$. 
Additional subgroup analyses were done according to the AL (Table 2) and the Km (Table 3) of the eyes. Long eyes were defined as AL longer than $25.5 \mathrm{~mm}$, short eyes were defined as AL shorter than $22.5 \mathrm{~mm}$, and eyes with $\mathrm{AL}$ measurements between them were defined as medium eyes. In the long eyes group, the mean $\mathrm{AL}$ measurement with Lenstar LS900 was significantly longer than with the other two devices, IOL Master 500 and IOL Master $700^{\circ}$ by mean differences of $0.047 \pm 0.03 \mathrm{~mm}(p<0.001)$ and 0.051 $\pm 0.06 \mathrm{~mm}(p=0.002)$, respectively. However, in the medium and short eyes groups, AL measurements among the three devices were not significantly different. Eyes with steep $\mathrm{Kms}$ were defined as those with $\mathrm{Km}$ values steeper than 46 diopters (D), eyes with flat Kms had values flatter than $42 \mathrm{D}$, and eyes with $\mathrm{Km}$ between the two were defined as medium $\mathrm{Km}$. In the medium $\mathrm{Km}$ subgroup, the IOL Master 500 measured significantly steeper than other two devices, while the steep $\mathrm{Km}$ and flat Km subgroups showed no significant differences. In the medium Km subgroup, the mean differences between IOL Master 500 were $0.072 \pm 0.22 \mathrm{D}(p=0.003)$ and $0.069 \pm 0.17 \mathrm{D}(p<0.001)$ with the IOL Master 700 and Lenstar LS900, respectively.

The Bland-Altman plots of agreement between each of the two devices for AL, Km, and ACD are shown in Fig. 1A-1I. All plots showed high agreement and narrow 95\% limits of agreement for the values of ocular parameters with each of the two devices.

For the 54 eyes that underwent cataract surgery and were implanted with ZCB00 (Tecnis), all had refractive PE within $1.5 \mathrm{D}$. Considering the mean $\mathrm{PE}$, the postoperative refraction was slightly hyperopic using the Haigis formula with IOL Master $500(0.05 \pm 0.46 \mathrm{D})$ and myopic for SRK/ $\mathrm{T}$ with both the IOL Master 700 and Hill-RBF formula with Lenstar LS900 $(-0.17 \pm 0.41$ and $-0.12 \pm 0.40 \mathrm{D}$, respectively) compared with the predicted value. However, there were no significant differences between the absolute PE predicted by three formulae with the three devices (Table 4).

\section{Discussion}

Cataract surgery is one of the most frequently performed and successful ophthalmic surgical procedures in recent decades. Therefore, many studies and developments regarding ocular biometry measurements and IOL power calculation have been performed to attain the desired postoperative refraction.

In this study, AL measurement failed in 12 eyes (9.7\%) using the IOL Master 500, three eyes (2.4\%) failed using the IOL Master 700 , and five eyes (4.0\%) failed using the Lenstar LS900; all of the failed eyes were from patients over 70 years old whose nuclear scores by LOCS III were over 3, except for three eyes with PSC. Hirnschall et al. [8] reported that $6.4 \%$ of eyes were not measured successfully with IOL Master 500 using partial coherence interferometry technology, and $91.3 \%$ of them were measurable with IOL Master 700, with a total failure rate of only $0.5 \%$. Buckhurst et al. [9] reported that the Lenstar LS900 was unable to take measurements due to dense media opacities in a similar number of patients to the IOL Master 500 for $9 \%$ to $10 \%$ of eyes. Although a small difference may exist in the acquisition rate according to the severity of cataracts in the study population, our study showed the same tendencies as the previous studies. In PSC, the opacities are located nearer to the nodal point of the lens, and more light rays interfere, which is one reason for measurement failures in devices based on interferometry and reflectometry [10]. Akman et al. [4] reported that using SS-OCT technology may overcome these problems, and it could be a primary reason for the higher acquisition rate for AL measurements with IOL Master 700 in the presence of PSC. Our study also showed that IOL Master 700 was advantageous in terms of measuring the AL, particularly for eyes with PSC.

In measuring the AL, Arriola-Villalobos et al. [11] reported that there was no significant difference in AL measurements when using the Lenstar LS900 or the IOL Master 700, while other studies demonstrated significantly longer AL measurements of Lenstar LS900 than IOL Master 500 [9,12-14]. Our results showed that Lenstar LS900 measured longer AL than IOL Master 500 and IOL Master 700. Olsen [15] noted that the measurement of AL constitutes the largest source of error in IOL calculation. Therefore, we performed a subgroup analysis of the AL to determine whether the difference between the measurements of the three devices was affected by the AL. Subgroup analysis revealed that Lenstar LS900 measured longer AL than IOL Master 500 and IOL Master 700 only in the long eyes. Lenstar LS900 may be more influenced by the media factor since it uses the principle of reflectometry through the medium of the object. The IOL Master 500 uses the wave- 
A
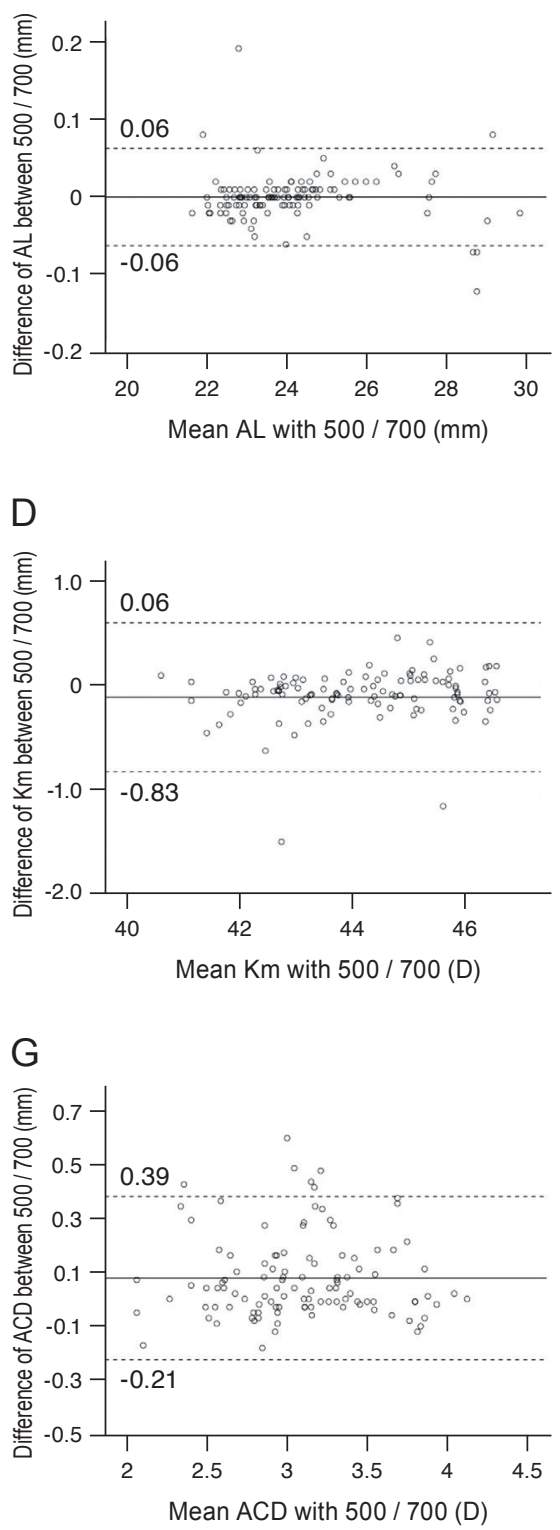

B

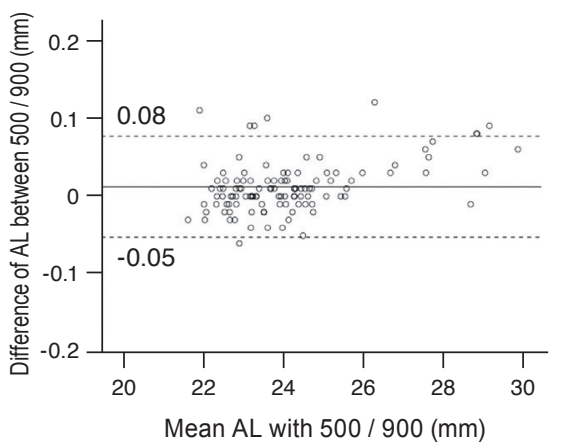

E

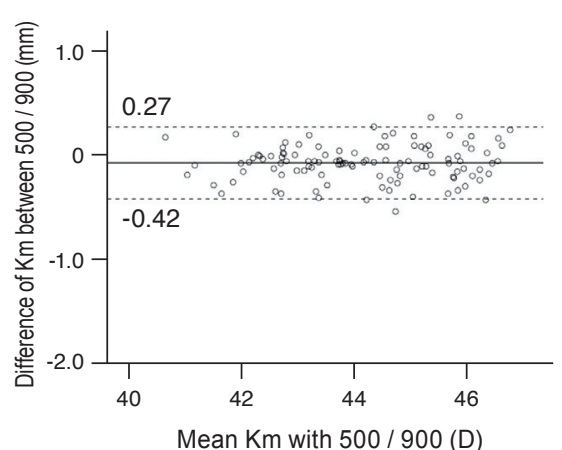

$\mathrm{H}$

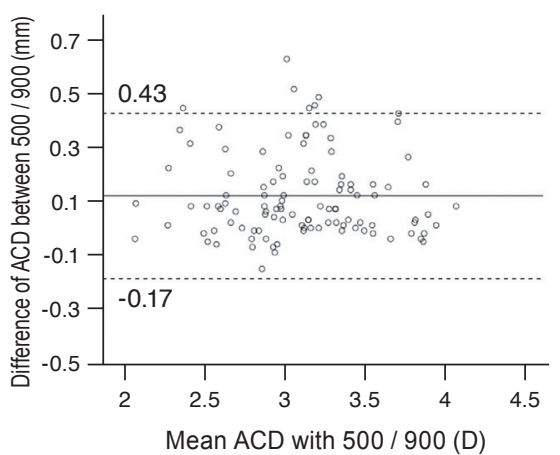

C

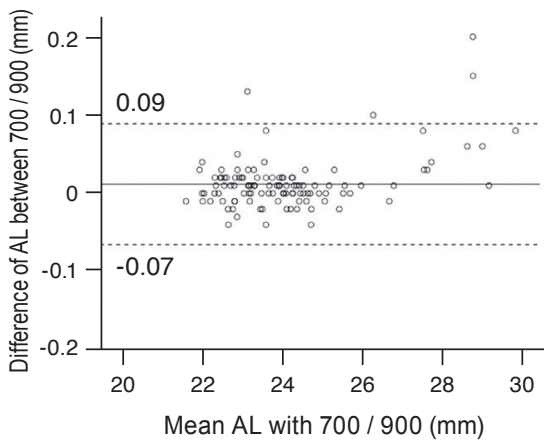

F

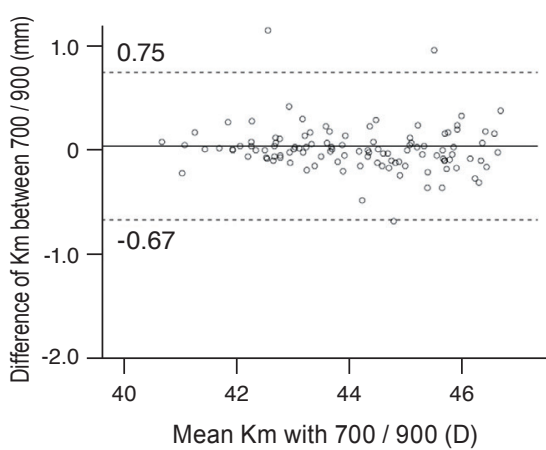

I

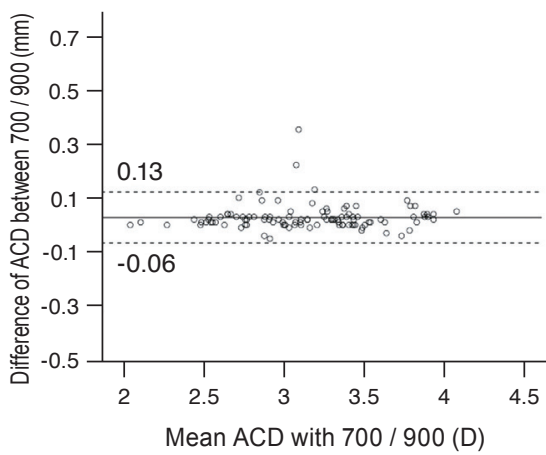

Fig. 1. A Bland-Altman plot showing the agreement of the ocular biometry measurements among three different devices. The middle solid line shows the mean difference, and the top and bottom dashed lines show the upper and lower 95\% limits of agreement, respectively. (A-C) Axial length (AL), (D-F) mean keratometry (Km), and (G-I) anterior chamber depth (ACD) between (A,D,G) IOL Master 500 and IOL Master 700, (B,E,H) IOL Master 500 and Lenstar LS900, and (C,F,I) IOL Master 700 and Lenstar LS900. D = diopters.

length of the $760 \mu \mathrm{m}$ infrared ray as the light source [16], the IOL Master 700 uses a 1,050 nm wavelength laser, and the Lenstar LS900 uses a 820-nm superluminescent diode laser. The difference in the transmittance of the wavelength due to the turbidity of the medium and the error caused by the increase in the length of the measurement object are combined. Also, the fact that each device uses its own adjustment algorithms to match with the ultrasonic measurements might cause slight differences in AL measurements. Consequently, in long eyes, it should be taken into account that the $\mathrm{AL}$ would be measured considerably longer when using Lenstar LS900, and since it is not known which value is actually true, further consideration is necessary to compare the actual prediction error substituted with the values measured by each device.

In the measurement of $\mathrm{Km}$, our result showed that IOL 
Table 4. Comparison of PEs among three distinct formulae of the built-in software of IOL Master 500, IOL Master 700, and Lenstar LS900

\begin{tabular}{|c|c|c|c|c|c|c|}
\hline & \multicolumn{3}{|c|}{$\mathrm{PE}(\mathrm{D})$} & \multicolumn{3}{|c|}{ Eyes within (\%) } \\
\hline & Mean PE & Absolute PE & Range & $0.5 \mathrm{D}$ & $1.0 \mathrm{D}$ & $1.5 \mathrm{D}$ \\
\hline IOL Master 500 & $0.05 \pm 0.46$ & $0.38 \pm 0.26$ & -0.88 to 1.08 & 72.2 & 96.3 & 100 \\
\hline IOL Master 700 & $-0.17 \pm 0.41$ & $0.35 \pm 0.28$ & -1.25 to 0.64 & 77.8 & 94.4 & 100 \\
\hline Lenstar LS900 & $-0.12 \pm 0.40$ & $0.35 \pm 0.24$ & -0.96 to 1.03 & 79.6 & 96.3 & 100 \\
\hline
\end{tabular}

Values are presented as mean \pm standard deviation if not otherwise indicated; Haigis formula for IOL Master 500, SRK/T for IOL Master 700, and Hill-RBF for Lenstar LS900 were used to calcuate.

$\mathrm{PE}=$ prediction error; $\mathrm{D}=$ diopters.

Master 500 measured steeper Km compared with IOL Master 700 and Lenstar LS900. Previously, Hoffer et al. [12] and Epitropoulos [17] reported that the Lenstar LS900 measured flatter $\mathrm{Km}$ than IOL Master 500 and Akman et al. [4] reported that IOL Master 700 measured flatter $\mathrm{Km}$ than IOL Master 500, which was consistent with our study. In subgroup analysis, the IOL Master 500 measured the steepest $\mathrm{Km}$ measurements among all the steep, medium, and flat $\mathrm{Km}$ subgroups; however, the difference was significant only in the medium Km subgroup. Differences in the sample size among the subgroups might have affected the significance. We found that the tendency of mean $\mathrm{Km}$ differences among the three instruments did not change with keratometry values. Both IOL Master 500 and IOL Master 700 use a telecentric method to measure the curvature of the cornea by projecting a light source onto the cornea. The IOL Master 500 uses six points $2.5 \mathrm{~mm}$ apart from the center of cornea, while the IOL Master 700 uses a total of 18 to 6 points at each, $1.5 \mathrm{~mm}, 2.4 \mathrm{~mm}$, and $3.2 \mathrm{~mm}$ from the center of the cornea [18]. The Lenstar LS900 uses 32 points using the dual zone keratometry method. It can be inferred that these differences in measurement methods have caused a slight difference in the keratometry results.

For ACD, the IOL Master 700 detects the longitudinal section of the eye by SS-OCT to measure the ACD, while the IOL Master 500 measures the distance between the cornea and the lens by obliquely illuminated slit lamp source. The Lenstar LS900 measures ACD when measuring several dimensions in a single step. Differences in these measurement principles and methods would have resulted in differences in the ACD measurement in our study that showed shallower ACD with the IOL Master 500 compared with the IOL Master 700 and Lenstar LS900. Previous studies also reported that reported that the Lenstar LS900 measures a deeper ACD than IOL Mas- ter $500[9,12,17]$. However, in comparing the IOL Master 500 and IOL Master 700, Akman et al. [4] reported that the IOL Master 700 measured shorter ACD with clinical significance, while Srivannaboon et al. [19] reported that the IOL Mater 700 measured longer ACD but with no significant difference compared to the IOL Master 500.

The results of the Bland-Altman plots to visualize and quantify the agreements of three main measurement outcomes by the IOL Master 500, IOL Master 700, and Lenstar LS900 revealed that, although statistically significant differences were observed, ocular biometry measurements by the three devices showed high agreement and narrow $95 \%$ limits of agreement. Moreover, there were no significant differences for the predictability of the refractive outcomes after cataract surgery among the three devices.

There were several limitations in our study. First, as our study population is composed of mixed cataracts, we could not compare the success rate of AL measurement according to the specific class of cataract. However, this reflects the real-world clinical setting, and the result was consistent with the previously reported success rates of each device. Second, refractive outcome was compared with different formulae using the built-in software of each device. However, by diversifying the formula, the validity of the three formulae, Haigis, SRK/T, and Hill-RBF can be identified through the result that there was no difference in predictability of refractive error between devices with high agreement. Furthermore, we were able to validate that Hill-RBF, which was recently introduced and is easily accessible with Lenstar LS900, shows comparable predictivity with both the Haigis and SRK/T formulae.

In summary, our study shows that the IOL Master 700 and Lenstar LS900 demonstrated superior acquisition rates of biometric measurements compared with the IOL Master 500. Ocular biometry by 3 different devices showed high 
agreement, although statistically significant differences were observed, and since there was no difference in predicting the refractive outcomes, those differences are clinically negligible.

\section{Conflict of Interest}

No potential conflict of interest relevant to this article was reported.

\section{References}

1. Fontes BM, Fontes BM, Castro E. Intraocular lens power calculation by measuring axial length with partial optical coherence and ultrasonic biometry. Arq Bras Oftalmol 2011;74:166-70.

2. Shammas HJ, Chan S. Precision of biometry, keratometry, and refractive measurements with a partial coherence interferometry-keratometry device. J Cataract Refract Surg 2010;36:1474-8.

3. Vogel A, Dick HB, Krummenauer F. Reproducibility of optical biometry using partial coherence interferometry : intraobserver and interobserver reliability. $J$ Cataract $R e$ fract Surg 2001;27:1961-8.

4. Akman A, Asena L, Gungor SG. Evaluation and comparison of the new swept source OCT-based IOLMaster 700 with the IOLMaster 500. Br J Ophthalmol 2016;100:1201-5.

5. Cruysberg LP, Doors M, Verbakel F, et al. Evaluation of the Lenstar LS 900 non-contact biometer. $\mathrm{Br} J$ Ophthalmol 2010;94:106-10.

6. Olsen T. Calculation of intraocular lens power: a review. Acta Ophthalmol Scand 2007;85:472-85.

7. Chylack LT Jr, Wolfe JK, Singer DM, et al. The lens opacities classification system III. The longitudinal study of cataract study group. Arch Ophthalmol 1993;111:831-6.

8. Hirnschall N, Varsits R, Doeller B, Findl O. Enhanced penetration for axial length measurement of eyes with dense cataracts using swept source optical coherence tomogra- phy: a consecutive observational study. Ophthalmol Ther 2018;7:119-24.

9. Buckhurst PJ, Wolffsohn JS, Shah S, et al. A new optical low coherence reflectometry device for ocular biometry in cataract patients. Br J Ophthalmol 2009;93:949-53.

10. McAlinden C, Wang Q, Pesudovs K, et al. Axial length measurement failure rates with the IOLMaster and Lenstar LS 900 in eyes with cataract. PLoS One 2015;10:e0128929.

11. Arriola-Villalobos P, Almendral-Gomez J, Garzon N, et al. Agreement and clinical comparison between a new sweptsource optical coherence tomography-based optical biometer and an optical low-coherence reflectometry biometer. Eye (Lond) 2017;31:437-42.

12. Hoffer KJ, Shammas HJ, Savini G. Comparison of 2 laser instruments for measuring axial length. $J$ Cataract Refract Surg 2010;36:644-8.

13. Holzer MP, Mamusa M, Auffarth GU. Accuracy of a new partial coherence interferometry analyser for biometric measurements. Br J Ophthalmol 2009;93:807-10.

14. Rohrer K, Frueh BE, Walti R, et al. Comparison and evaluation of ocular biometry using a new noncontact optical low-coherence reflectometer. Ophthalmology 2009;116:208792.

15. Olsen T. Sources of error in intraocular lens power calculation. J Cataract Refract Surg 1992;18:125-9.

16. Santodomingo-Rubido J, Mallen EA, Gilmartin B, Wolffsohn JS. A new non-contact optical device for ocular biometry. Br J Ophthalmol 2002;86:458-62.

17. Epitropoulos A. Axial length measurement acquisition rates of two optical biometers in cataractous eyes. Clin Ophthalmol 2014;8:1369-76.

18. Hoffer KJ, Hoffmann PC, Savini G. Comparison of a new optical biometer using swept-source optical coherence tomography and a biometer using optical low-coherence reflectometry. J Cataract Refract Surg 2016;42:1165-72.

19. Srivannaboon $\mathrm{S}$, Chirapapaisan $\mathrm{C}$, Chonpimai $\mathrm{P}$, Loket $\mathrm{S}$. Clinical comparison of a new swept-source optical coherence tomography-based optical biometer and a time-domain optical coherence tomography-based optical biometer. J Cataract Refract Surg 2015;41:2224-32. 